\title{
Origin of the left coronary artery from the right pulmonary artery and ventricular septal defect in a child of a mother with raised plasma phenylalanine concentrations throughout pregnancy
}

\author{
D Henglein, $H$ Niederhoff, $H$ Bode
}

\begin{abstract}
A child with anomalous origin of the left coronary artery from the right pulmonary artery, ventricular septal defect, fetal growth retardation, and facial abnormalities was born to a woman in whom plasma phenylalanine concentrations had been raised throughout pregnancy. The cardiac abnormalities were diagnosed by angiography when the child was eight months old. The anomalous coronary artery was imaged in a subsequent echocardiogram. Development retardation was caused by maternal phenylketonuria, which may also have been responsible for the development of the ventricular septal defect and the coronary anomaly. If dietary treatment of the mother had been started before pregnancy damage to the child might have been prevented.
\end{abstract}

Anomalous origin of the left coronary artery from the pulmonary artery as an isolated lesion usually presents as myocardial ischaemia. ${ }^{1}$ Haemodynamic features caused by associated intracardiac lesions may obscure the clinical features of the coronary artery anomaly. Reports of children with this anomaly and an associated ventricular septal defect show that the coronary anomaly was not detected until operative closure of the ventricular septal defect was followed by a fatal outcome or until signs of myocardial ischaemia developed in children in whom the ventricular septal defect had spontaneously become smaller.

We report on one infant in whom the anomaly-origin of the left coronary artery from the right pulmonary artery-was diagnosed before operation. We know of five reports of this rare condition..$^{2-6}$ The mother of the patient we describe was found to have raised plasma concentrations of phenylalanine after delivery.

\section{Case report}

The female infant was delivered by caesarean section at 36 weeks' gestation because of slowing of the fetal heart rate. An ultrasonic evaluation in week 15 of pregnancy had shown growth retardation of the fetus. Apart from this, pregnancy had been uneventful. There was no history of congenital heart disease in the family.
At birth the child was small for dates (birth weight of $1460 \mathrm{~g}$ ), microcephalic, and showed several signs of facial dysplasia consistent with the high maternal plasma concentration of phenylalanine $(2.46-3.06 \mathrm{mmol} / \mathrm{l})$ measured soon after delivery. In the next few weeks a grade $3 / 6$ continuous systolic murmur was audible along the infant's left sternal border. A low frequency mid-diastolic murmur could be heard at the apex of the heart. The intensity of the second part of the second heart sound was increased. The liver was slightly enlarged, there was no cyanosis, and the peripheral pulses were normal. Follow up chest $x$ rays and electrocardiograms showed cardiomegaly, increased pulmonary vascularity, and signs of double ventricular hypertrophy without $T$ wave inversion or ST segment or $T$ wave anomalies. Echocardiographic examination showed a ventricular septal defect (diameter $4 \mathrm{~mm}$ ), left and right ventricular and septal hypertrophy, and slight atrial enlargement. The shortening fraction and ejection fraction, calculated by means of $M$ mode echocardiography, were within normal limits. During the ensuing months there was no clinical or electrocardiographic evidence of ischaemia of the myocardium.

When the child was eight months old cardiac catheterisation was performed. She was small $(6.1 \mathrm{~kg})$-in the third percentile for weight at eight months. Cardiac catheterisation confirmed the diagnosis of ventricular septal defect. Pulmonary artery pressure was raised because the left and right ventricular systolic pressures were almost equal. The calculated left to right shunt was $57 \%$ and pulmonary vascular resistance was within normal limits. Cineangiography provided the complete diagnosis. Injection into the left ventricle showed normal contractility of this chamber, no mitral valve incompetence, and a ventricular septal defect in the typical high location. After left ventricular contraction the aortic root, the right coronary artery, and a relatively large conal branch were seen without any evidence of left coronary artery filling. Injection into the right ventricle and selective angiography of the right pulmonary artery showed the origin of the anomalous left coronary artery on the right pulmonary artery approximately $1 \mathrm{~cm}$ away from the bifurcation (fig 1). The anomalous artery ran below and behind the pulmonary trunk. Then it divided. One of the branches
Correspondence to Dr D Henglein, 22 Rue Monsieur le Prince, 75006 Paris, France. 
Figure 1 Angiograms after injection of contrast into the right pulmonary artery showing the anomalous origin of the left coronary artery from the right pulmonary artery in (a) frontal and (b) lateral views.

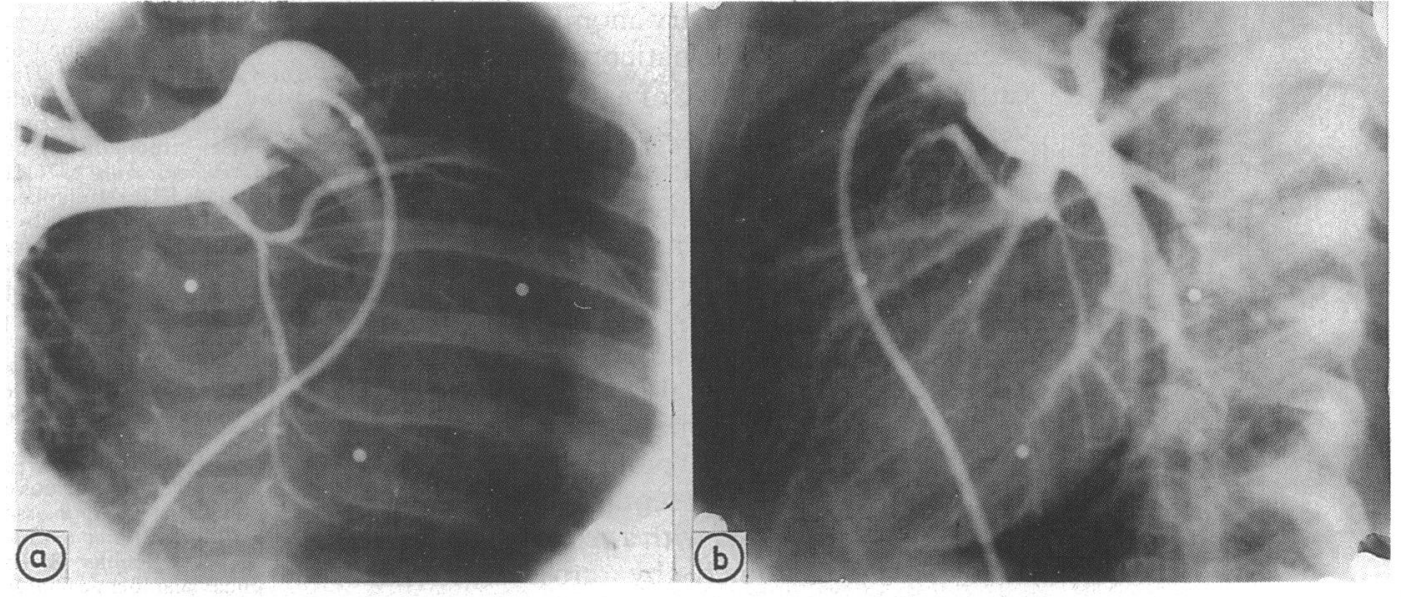

followed the usual course of the anterior descending coronary artery and the other that of the circumflex coronary artery. No collaterals were seen between the left and right coronary artery system.

This coronary anomaly was examined by cross sectional echocardiographic study through the aortic root. In the short axis view no coronary artery ostium was seen in the left aortic sinus. Slight cranial angulation of the transducer showed the anomalous coronary artery following a straight course below and to the left behind the ascending aorta without any contact with the aortic sinus (fig 2).

At the age of 14 months the child was in good physical condition without congestive cardiac failure. Tests showed developmental retardation of about two months. The head circumference was consistently about $2 \mathrm{~cm}$ below the third percentile for corresponding age and there were no signs of a decrease in the left to right shunt or of myocardial ishaemia. An operation was performed to reimplant the left coronary artery into the ascending aorta and close the perimembranous ventricular septal defect with a patch. When the aorta was unclamped ventricular activity resumed immediately without electrocardiographic evidence of myocardial ischaemia. Bypass was stopped under stable circulatory conditions. However, one hour later the blood pressure suddenly dropped and the child died despite resuscitative measures. The parents did not agree to necropsy.

ALC $A$, left atrium, coronary artery.

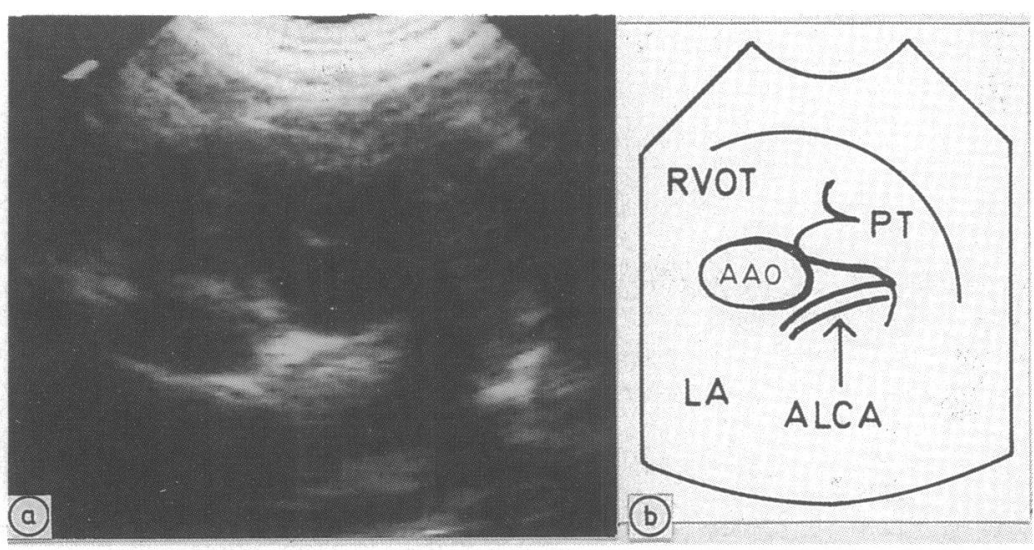

\section{Discussion}

The cardiac malformation in this infant consisted of an anomalous origin of the left coronary artery from the right pulmonary artery and a ventricular septal defect. These anomalies, the growth retardation before and after birth, the microcephaly, and the abnormal facial appearance of this newborn infant suggested that maternal phenylketonuria might be the common cause. ${ }^{7}$ Grossly raised maternal plasma concentrations of phenylalanine soon after delivery confirmed the diagnosis. If maternal dietary treatment had been started before conception ${ }^{910}$ this child might not have been damaged.

To date there have been five reports of origin of the left coronary artery from the right branch of the pulmonary artery..$^{2-6}$ In our patient the left coronary artery originated at exactly the same site as discussed by other workers - that is $0.5-1 \mathrm{~cm}$ distal to the bifurcation from the right pulmonary artery. In the two cases reported by Doty et al and Hamilton et al there were no associated cardiac lesions. ${ }^{23}$ Surgical correction was successful by anastomosis of the left coronary artery to the right subclavian artery in one child ${ }^{2}$ and by implantation of the anomalous coronary artery into the aorta in the other. ${ }^{3}$ Haemodynamic changes resulting from an associated ventricular septal defect obscured the clinical features of the coronary anomaly in the cases reported by Rao et al and by Wilcox $e t$ al. ${ }^{56}$ Both children died immediately after surgical closure of the ventricular septal defect. Necropsy showed the associated anomalous origin of the left coronary artery from the right pulmonary artery. The haemodynamic basis for this fatal outcome was the drop in pulmonary arterial pressure when the ventricular septal defect was closed. This caused a fall in coronary artery perfusion followed by myocardial ischaemia and death. The same mechanism was seen in two children reported by Pinsky et al $^{11}$ : spontaneous reduction in the size of a ventricular septal defect resulted in myocardial ischaemia and infarction. Earlier identification and repair of the anatomical abnormality could have prevented the irreversible myocardial damage in all of these reported patients. 
The associated coronary anomaly could have been identified in our patient during cardiac catheterisation at the age of eight months. Left ventricular angiography clearly showed the right coronary artery but not the left coronary artery. This led to the suspicion of a coronary artery anomaly, which was confirmed and identified by right ventricular and right pulmonary angiography. A cross sectional echocardiogram after cardiac catheterisation also identified the anomalous coronary artery. So the identification of anomalous origin of the left coronary from the pulmonary artery in patients with ventricular septal defect would be increased if every child with a ventricular septal defect was examined by cross sectional echocardiography to assess the origin of the coronary arteries from the aortic root, ventricular cineangiography to image the arrangement of the coronary arteries, and if necessary by aortography.

In addition, we have started a routine assessment of coronary arteries by echocardiography in every child in our outpatient clinic. We are, however, aware that rare anomalies such as an origin of an accessory coronary artery from the pulmonary artery ${ }^{12}$ or origin of the anterior branch of the left coronary artery from the pulmonary artery ${ }^{13}$ will not be excluded by this method.
1 Askenazi J, Nadas A. Anomalous left coronary artery originating from the pulmonary artery: report on 15 cases. Circulation 1975;51:976-87.

2 Doty DB, Chandramouli B, Schicken RE, Lauer RM, Ehrenhaft JL. Anomalous origin of the left coronary artery from the right pulmonary artery. $J$ Thorac Cardiovasc Surg 1976;71:787-91.

3 Hamilton JRL, Mulholland HC, O'Kane HOJ. Origin of the left coronary artery from the right pulmonary artery: a Thorac Surg 1986;41:446-8.

4 Masel LF. Tetralogy of Fallot with origin of the left coronary artery from the right pulmonary artery. Med $J$ Aust 1960;i:213-7.

5 Rao BNS, Lucas RV, Edwards JE. Anomalous origin of the left coronary artery from the right pulmonary artery left coronary artery from the right pulmonary artery
associated with ventricular septal defect. Chest associated with

6 Wilcox WD, Hagler DJ, Lie JT, Danielson GK, Smith HC, Fulton RE. Anomalous origin of left coronary artery from pulmonary artery in association with intracardiac lesions. $J$ Thorac Cardiovasc Surg 1979;78:12-20.

7 Lipson A, Beuhler B, Bartley J et al. Maternal hyperphenylalaninemia fetal effects. J Pediatr 1984;104:216-20

8 Lenke RR, Levy HL. Maternal phenylketonuria and hyperphenylalaninemia. An international survey of the outcome of untreated and treated pregnancies. $N$ Engl $J$ Med 1980;303:1202-8.

9 Bode H, Urbanek R, Henglein D, Niederhoff $H$. Embryofetopathie infolge einer postnatal erkannten maternalen Phenylketonurie Helv Paediatr Acta 1987;42:463-9.

10 Nielson KB, Wamberg E, Weber J. Successful outcome of pregnancy in a phenylketonuric woman after low phenylpregnancy in a phenylketonuric woman after low phenyl1979;i:1245.

11 Pinsky W, Gilette P, Duff D, et al. Anomalous origin of left coronary artery from the pulmonary artery with ventricular septal defect. Circulation 1978;57:1026-30.

12 Krause W. Uber den Ursprung einer accessorischen A coronaria cordis aus der A. pulmonalis. Z Rat Med 1865;24:225.

13 Probst $P$, Pachinger $O$, Koller $H$, Niederberger $M$, Kaind F. Origin of anterior branch of left coronary artery from pulmonary trunk. Br Heart J 1976;38:523-5. 